\title{
DISTRIBUTED SYSTEM SUPERVISION AND CONTROL WITH myRIO AND OPC-UA
}

\author{
Francisco Blanes Noguera \\ pblanes@ai2.upv.es \\ Antonio Correcher Salvador \\ ancorsal@ai2.upv.es \\ Jose Antonio Manzano Alcaide \\ jomanal@posgrado.upv.es \\ Andrés Benlloch Faus \\ anbenfau@ai2.upv.es
}

Instituto Universitario de Automática e Informática Industrial

Universitat Politécnica de Valencia

\section{Resumen}

In this project tackle the design, supervision and control of a pilot plant, which incorporate several renewable energy sources to simulate control strategies which satisfy electricity demand.

Tha main characteristic about the work consist in using two different environment, on one hand, the hardware myRIO that control the plant and manage energy sources. On the other hand, supervision system is based on OPC-UA software multiplatform, which provides interoperability and standarization.

Palabras clave: Process supervision and control, OPC-UA protocol, distributed control, renewable energy.

\section{INTRODUCCIÓN}

En este trabajo se diseñará una planta piloto de energías renovables, en la que se montará cada fuente con la instrumentación necesaria para la producción de energía. También se incluye varios circuitos para simular diferentes condiciones meteorológicas.

La planta piloto tendrá un sistema de adquisición de datos basado en la NI myRIO y un sistema de control con diferentes modos a implementar para satisfacer una demanda de energía.

Para la parte de supervisión se creará una aplicación siguiendo el modelo cliente-servidor con comunicación OPC-UA (OPC Unified Architecture) que permitirá la interacción entre el sistema myRIO y otro PC conectado desde cualquier lugar, en el cual se encontrará el SCADA diseñado para la supervisión. De esta forma, se crea un sistema distribuido que permite un control en tiempo real, al tiempo que se relega la supervisión del sistema a la aplicación del PC.

\subsection{Motivación}

"Los combustibles fósiles no están hechos por el hombre, no pueden ser reciclados. Cuando se terminen, ¡Se habrán terminado para siempre!” [2].

Cada vez es más necesario la creación de nuevas soluciones capaces de suministrar energías durante todo el día y en todas las épocas del año, mediante la combinación de energías renovables, con el objetivo de en un futuro sustituir las plantas de energías contaminantes las cuales se están agotando. Por eso, es interesante la realización de un proyecto sobre energías renovables que pretende combinarlas y gestionarlas para mejorar la demanda de energía.

El trabajo se diseñará lo más parecido posible a los sistemas industriales típicos, con un sistema distribuido controlado con OPC-UA, ya que este sistema está cada vez más implantado en la industria y permite una comunicación segura y operativa. Además, al usar el OPC-UA en lugar del OPC clásico permite emplear el sistema de supervisión con distintos sistemas operativos por lo que aumenta la funcionalidad de la planta, creando un sistema potente con posibilidad de crecimiento al ser compatible con las últimas tecnologías.

\subsection{Objetivos.}

Los objetivos que se pretenden alcanzar al final de este proyecto son:

1. Diseñar e implementar la instrumentación necesaria para la experimentación de una planta piloto de energías renovables.

2. Simular diferentes condiciones meteorológicas con el objeto de estudiar el comportamiento de la planta sobre estas 
condiciones, con una determinada demanda de energía.

3. Ser capaz de obtener los datos de la planta y hacer un control sobre el sistema empotrado NI myRIO para la gestión óptima de la energía generada por la planta.

4. Desarrollar un sistema distribuido mediante un modelo cliente-servidor OPC-UA, para la supervisión de la planta piloto sobre un SCADA.

\subsection{Estado del arte.}

Una de las principales características utilizadas en este trabajo es el protocolo de comunicaciones usado es el OPC-UA (OLE for Process Control - Unified Architecture). Esta tecnología fue creada para el control y visualización de datos en la automatización industrial, permite de manera fácil y segura el intercambio de información entre diversas plataformas, además de un flujo continuo de información entre dispositivos de diferentes proveedores. Hoy en día hay más de 4200 proveedores que han creado mas de 35000 productos diferentes. En el OPC-UA aglutina las funcionalidades clásicas del OPC (acceso a datos, históricos, alarmas, eventos), y permite una interoperabilidad y portabilidad de la que carece el OPC clásico [6].

Además, en este trabajo se ha hecho uso de un proyecto anterior, en el que se realizaba el estudio, control y adquisición de datos en la pila de hidrógeno [3].

\section{PLANTA PILOTO}

Para llevar a cabo el diseño, control y supervisión de la planta han sido necesarios varios elementos hardware que se mencionan a continuación.

\subsection{NI myRIO}

El dispositivo embebido de la Figura 1 es un sistema diseñado para estudiantes y consta de un microprocesador (basado en la arquitectura ARM Cortex-A9 dual-Core) una FPGA Xilinx y un conjunto de entradas/salidas programables (10 salidas analógicas, 6 salidas digitales y 40 líneas de E/S digital). Además, dispone de conectividad alámbrica e inalámbrica. Esta herramienta puede ser programada tanto en LabVIEW como en C/C++ [5].

En este trabajo, el módulo myRIO se programará mediante LabVIEW y realizarán las tareas de adquisición de datos y control de la planta.

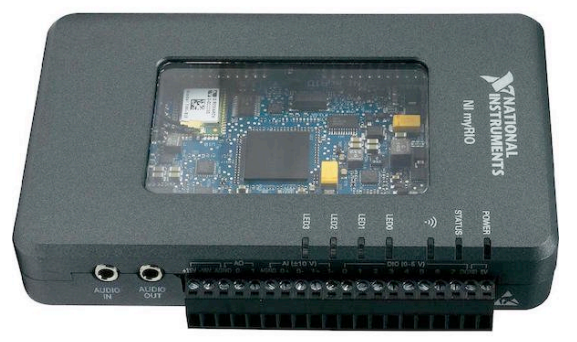

Figura 1: NI myRIO.

\subsection{Arduino UNO}

Es una placa con el aspecto de la Figura 2, basada en el microcontrolador ATmega328. Cuenta con 14 entradas/salidas digitales, de las cuales 6 se pueden utilizar como salidas PWM (Modulación por ancho de pulsos) y otras 6 son entradas analógicas [4].

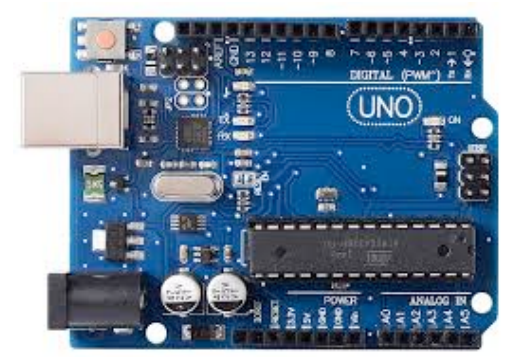

Figura 2: Arduino UNO.

Esta placa se encargará de simular las diferentes condiciones de sol y viento. Se comunicará con la myRIO a través del protocolo SPI. La myRIO le dirá las condiciones a simular, actuando como maestro.

\subsection{Aerogenerador}

Es un dispositivo que aprovecha la energía cinética del viento para transformarla en energía eléctrica por medio de sus hélices y de un alternador, el alternador transforma la energía mecánica de las hélices en eléctrica.

Las características del Aero usado en el proyecto depende del tipo y cantidad de hélices usadas, para dicho proyecto se usarán 6 hélices para aumentar la cantidad de energía proporcionada. Con esta configuración y una velocidad de $16 \mathrm{~km} / \mathrm{h}$ se podría llegar a obtener $750 \mathrm{rpm}$ con una tensión de $2.5 \mathrm{~V}$ y $50 \mathrm{~mA}$. 


\subsection{Célula fotovoltaica}

Células solares o células fotovoltaicas son pequeños elementos fabricados de un material semiconductor cristalino dopado, este dispositivo transforma directamente la energía solar en energía eléctrica por el efecto fotovoltaico (la luz solar transporta energía en forma de fotones, cuando estos inciden en la célula solar y son absorbidos provocan una corriente eléctrica). [1]

Para el diseño de la planta piloto se utilizan dos células fotovoltaicas de $5 \mathrm{~V}$ y $250 \mathrm{~mA}$ conectadas en paralelo como el resto de las fuentes de energía.

\subsection{Pila de hidrógeno y electrolizador}

Para la utilización del hidrógeno como fuente de energía serán necesario dos dispositivos:

Electrolizador: consta de dos electrodos fabricados de material conductor que al sumergirlos en un medio líquido (electrolito), y al aplicar una diferencia de potencial genera una reacción electroquímica mediante la que el electrolito se descompone en varios productos(electrólisis). En la electrólisis del agua, se descompone en oxígeno e hidrógeno, el cual se puede almacenar para después usarlo en la pila de hidrógeno.

Pila de hidrógeno: es un sistema electroquímico que convierte la energía química directamente en energía eléctrica. Consta de dos electrodos uno negativo y otro positivo, separados por un electrolito. El hidrógeno alimenta el ánodo mientras que el oxígeno alimenta el cátodo.

En el proyecto se usará un electrolizador que necesita un voltaje de entrada entre $1,8 \mathrm{~V}$ y $3 \mathrm{~V}$ a $0,7 \mathrm{~A}$ para producir hidrógeno. El tipo de pila usada es pila de membrana polimérica (PEM) cuyo voltaje de salida medio es de $0,6 \mathrm{~V}$ con una intensidad de $360 \mathrm{~mA}$ produciendo $210 \mathrm{~mW}$ dependiendo de la carga colocada en la salida.

\subsection{Baterías}

Se usarán tanto baterías de ion de litio (Li-ion) de $3,7 \mathrm{~V}$ y una capacidad de $2300 \mathrm{mAh}$ como de $\mathrm{Ni}-\mathrm{Cd}$ de $1,2 \mathrm{~V}$ y una capacidad de $1900 \mathrm{mAh}$, lo que permite hacer un proceso de carga y almacenamiento de energía, aumentando la funcionalidad de la planta y pudiendo satisfacer los picos de demanda mediante el proceso de descarga.

\section{Desarrollo del proyecto}

Para el desarrollo del proyecto se ha divido el trabajo en cuatro partes de acuerdo con su funcionalidad como se indica en la Figura 3.

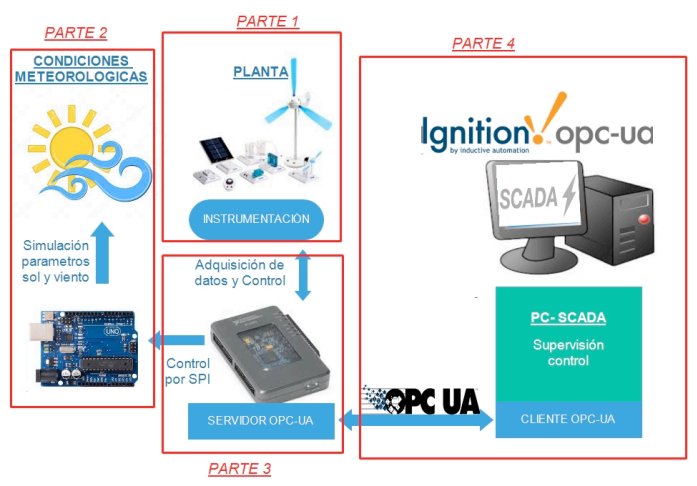

Figura 3: Esquema proyecto.

Todos los componentes de la planta (generadores de energía y consumidores) están conectadas al controlador principal, la myRIO, este realiza las tareas de adquisición de datos y control sobre la planta. Además, se encarga de pasar los datos a Arduino para que realice las simulaciones de sol y viento. Por último, la myRIO crea el servidor OPCUA con el objetivo de realizar la comunicación con otro ordenador por medio del cliente-servidor OPCUA para las tareas de supervisión.

\subsection{Diseño de la planta piloto}

Para poder hacer uso de la planta es necesario desarrollar la instrumentación necesaria para cada fuente de energía de forma integrada.

\subsubsection{Célula fotovoltaica}

Para el caso de la célula fotovoltaica se montará la estructura de la Figura 4, en la que se tiene un foco en un lado y en el otro lado se fijarán las dos células fotovoltaicas de forma que la luz del foco incida perpendicularmente a las células. Además, se incluirán en las células unos sensores de temperatura para evitar que se calienten en exceso.

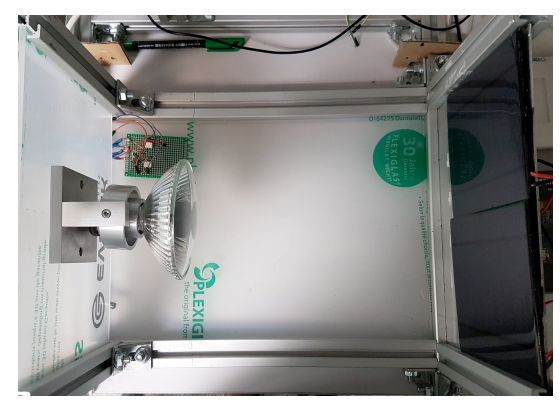

Figura 4: Estructura célula fotovoltaica. 


\subsubsection{Aerogenerador}

Para el aerogenerador se montará la estructura de la Figura 5.

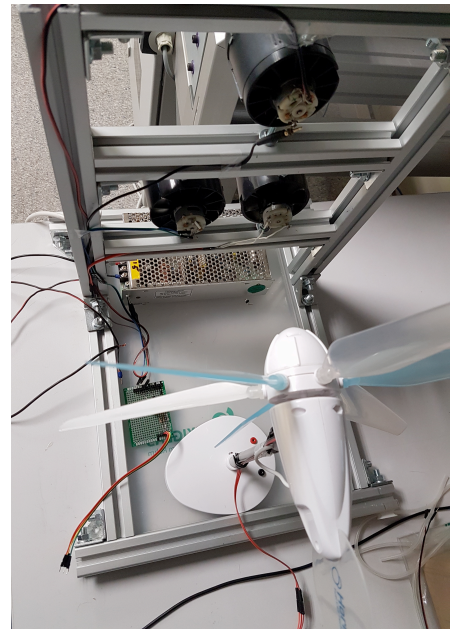

Figura 5: Estructura Aerogenerador.

Los perfiles de aluminio dejarán espacio para colocar los motores. Estos motores son los encargados de generar el viento necesario para mover el aerogenerador. Además, el aerogenerador dispondrá de un sensor de infrarrojo que se utilizará como encoder, para contar el número de vueltas y poder sacar las rpm a las que gira.

\subsubsection{Pila hidrógeno}

La detección del nivel de hidrógeno en el depósito se realiza por medio de un sistema de visión como el de la Figura 6, consta de una cámara en un entorno cerrado, para la adquisición de la imagen.

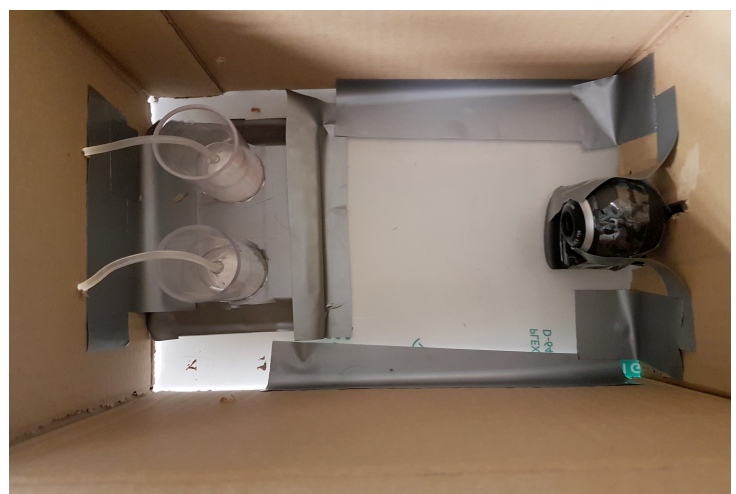

Figura 6: Sistema de visión depósito de hidrogeno.

La pila de hidrógeno y el electrolizador estarán conectados al almacenamiento de hidrógeno y oxígeno por medio de unos tubos y permitirán la producción de electricidad e hidrógeno según se requiera.

\subsubsection{PCB principal}

En este apartado se define la PCB principal del sistema, esta se encarga de conectar todas las fuentes de energía y las cargas. Los puertos de la myRIO estarán exportados en la PCB por medio de un cable paralelo y un conector en la PCB, las salidas digitales que controlan las fuentes y las cargas están aisladas por medio de optoacopladores, que separan las señales de control de la myRIO del circuito principal. Las señales de control se conectan a los transistores que activan/desactivan los generadores y consumidores de energía. La colocación de estos elementos en la PCB se muestra en la figura 7.

Esta PCB también incluye sensores de corriente y tensión para la lectura de datos.

-Sensor de corriente ACS712: consta de un sensor HALL de alta prestaciones junto con un canal de conducción, cuando la corriente fluye por el canal de cobre genera un campo magnético que es detectado por el sensor hall y es convertido en una tensión. Esta tensión es proporcional a la intensidad que atraviesa el sensor.

-Sensor de tensión MCP3008: Este dispositivo es un convertidor analógico-digital de aproximación sucesiva de 10bit con circuitería integrada de muestreo y retención. Se puede programar para proporcionar 4 pares de entradas (cada entrada con una tierra) u 8 salidas asimétricas (con una tierra común) la comunicación con el dispositivo será mediante el protocolo SPI.

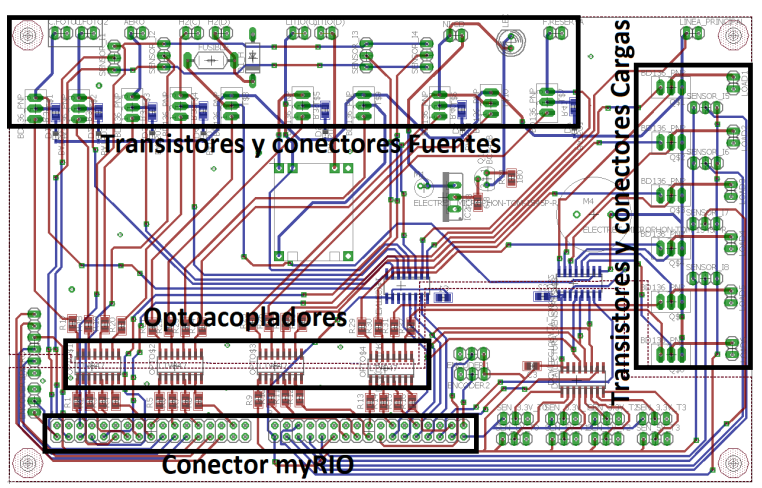

Figura 7: PCB principal.

\subsection{Simulación de las condiciones meteorológicas}

Para las simulaciones de la planta se introducirá unos valores de demanda de energía, de los que se pueden sacar de valores reales y escalarlo a un valor posible 
para la planta. Además, se pretende dar el valor de radiación y velocidad de viento introduciéndolos por el SCADA del programa ignition.

\subsubsection{Simulación del Sol}

Se diseña una PCB con un circuito de control de fase o control de ángulo de disparo, este regulará la intensidad de la luz para variar los valores de radiación del sol. Este circuito es el que aparece en la Figura 8 y se compone de dos partes [7].

-Detector de cruce por cero: sincroniza la parte de alterna con el controlador (Arduino), para ello el detector de cruce por cero detecta cuando la onda senoidal pasa por el cero. Se usa un optoacoplador cuyo fotoemisor estará encendido tanto en el semiciclo positivo como en el negativo de la señal de red, por lo que solo se apagará en el cruce por cero. Una entrada del Arduino estará conectada a tierra cuando el optoacoplador este activo, cuando la señal de red pasa por cero y el optoacoplador se desactiva, la entrada de Arduino deja de estar conectada a tierra y detecta $5 \mathrm{~V}$ provenientes de una resistencia de PULL-UP, con lo que el Arduino detecta el cruce por cero.

-Control de ángulo de disparo: Una vez se conoce el momento del cruce por cero, se puede regular la intensidad de la luz activándose una salida de Arduino y esta a su vez un TRIAC un tiempo $t$ después del momento de cruce por cero, así se limita la onda senoidal y se regula la tensión recibida por la bombilla.

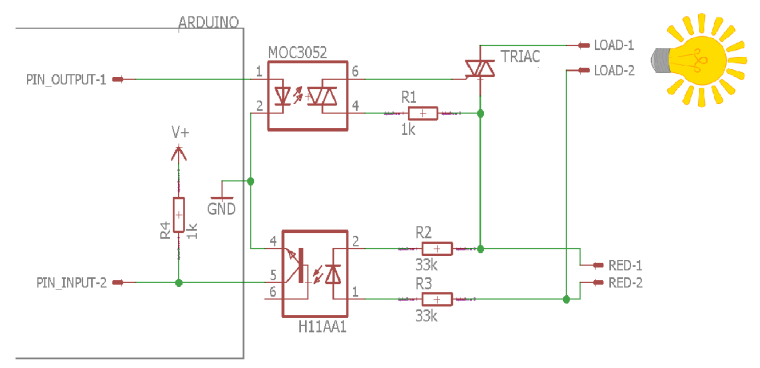

Figura 8: Circuito de control de fase.

\subsubsection{Simulación del viento}

Para la simulación del viento se utilizará otra PCB que controla la velocidad de los motores (DC).

En la PCB diseñada se implementará un circuito que usa una fuente de alimentación de $15 \mathrm{~V}$ para alimentar a los motores, la alimentación a los motores le llegará por medio de un transistor que estará trabajando en la región activa, por lo que la intensidad que le llega a los motores dependerá de la intensidad que le llegue a la base del transistor. La intensidad de base del transistor se controla a través del Arduino por medio de una salida PWM.

\subsection{Control de la planta}

El control se realiza mediante la myRIO y un programa en LabVIEW, el programa se dividirá en la parte de adquisición de datos, que se encarga de obtener todos los datos de la planta y en algunos casos de procesarlos (cálculo de rpm y procesamiento de imagen para obtener el nivel del depósito de hidrógeno).

La parte de control tendrá dos modos de actuación que se verán a continuación.

\subsubsection{Control en Manual}

En el control en manual se puede activar/desactivar cada fuente/carga por separado, será el supervisor el que se encargue de generar la electricidad y además servirá para realizar las pruebas y ajustes de la planta.

\subsubsection{Control en Auto}

Para el control en automático se utiliza un controlador Fuzzy para activar/desactivar las fuentes necesarias para satisfacer la demanda de energía requerida. El lazo de control del regulador será como el de la Figura 10.

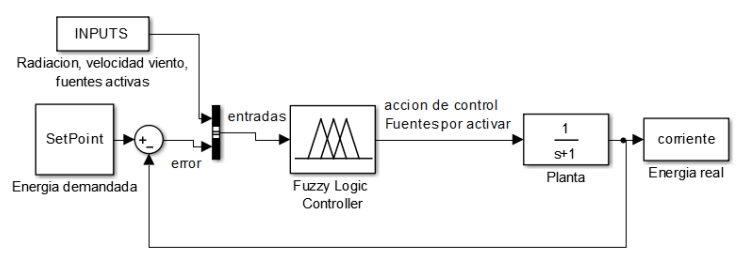

Figura 10: Lazo de control.

Se usará Fuzzy system designer de LabVIEW para diseñar un regulador proporcional difuso. La variable de entrada al regulador será la diferencia entre el SP de energía demandada y el valor de energía real que está proporcionando en ese momento la planta, que será el error. La variable de entrada al regulador difuso tendrá tres funciones de membresía las cuales son NEGATIVO (cuando la energía real es mayor a la demanda), ZERO (cuando la resta es cero o próximo a este) y POSITIVO (Cuando la energía demandada es mayor a la real). Con estas tres funciones de entrada se tendrá 3 salidas: quitar fuente, no hacer nada o añadir fuente. Que se pueden ver en la figura 11.

Para este regulador se tendrán 3 reglas muy simples, si la entrada es NEGATIVA se quita fuente, si es 
ZERO no se hace nada y si es POSITIVA se añade fuente.

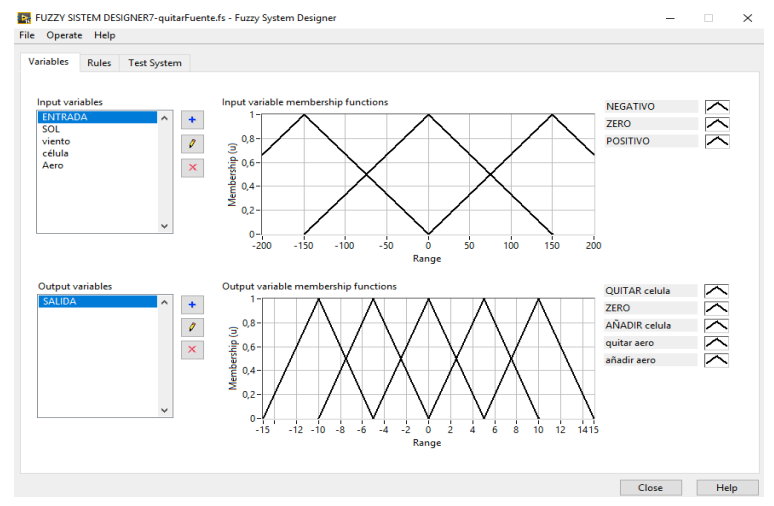

Figura 11: Fuzzy system designer.

Una vez configurado el regulador proporcional de forma sencilla se incluirán las entradas de radiación solar, velocidad del viento y las fuentes activadas para que el regulador ademas sea capaz de decir que fuente se va a activar en cada momento con el uso de las nuevas entradas y las reglas. Las reglas aumentarán considerablemente en complicación con el incremento del número de entradas.

Una vez hecho esto se creará un archivo que contiene los datos del control, este archivo se podrá cargar en LabVIEW con el programa de la Figura 12 e introduciéndole los valores de las entradas.

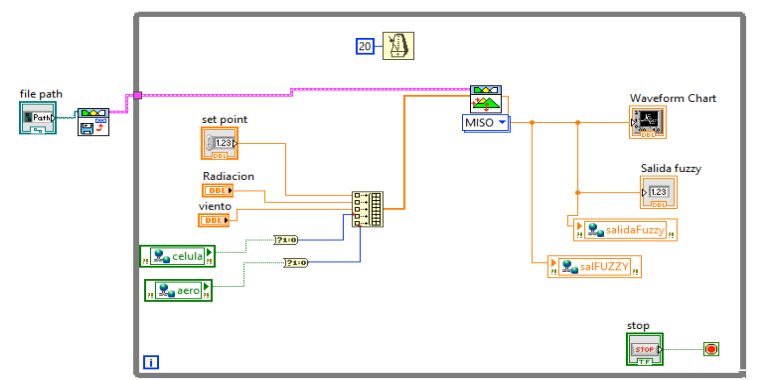

Figura 12: Implementación regulador en LabVIEW.

Con el valor de salida del regulador se filtrará entre unos rangos de valores y unas funciones lógicas para activar y desactivar la fuente que se necesite.

\subsection{Supervisión de la planta}

Actualmente en las plantas industriales se está progresivamente utilizando el protocolo OPC-UA. El protocolo OPC-UA se basa en el modelo clienteservidor y permite una comunicación continua desde los dispositivos de planta (PLC's, sistemas empotrados etc) hasta el sistema central de control o de supervisión.
Para realizar la supervisión de la planta se desplegará y configurará un servidor OPC-UA en el sistema empotrado myRIO y este nos permitirá acceder desde cualquier cliente a las variables que contiene.

Se creará un cliente OPC-UA desde otro PC con el software de ignition que se conectará al servidor OPC-UA en la placa myRIO, una vez con acceso desde ignition a las variables del servidor se le asignará un tag a cada variable para poder utilizarlas y se diseñará el SCADA para la supervisión y control de la planta.

El SCADA consta de 3 partes:

\subsubsection{Control de fuentes}

En esta pantalla se tendrá un esquema visual de las fuentes de energía conectadas a la PCB además de un apartado donde se puede seleccionar el modo de control, como ya se comentó que puede ser manual o automático. También se incluye una tabla y una gráfica para la visualización de las variables en la planta y una imagen que permite conocer la caracterización de las distintas fuentes de energía. La pantalla del SCADA que realiza todo esto es la que aparece en la Figura 13.

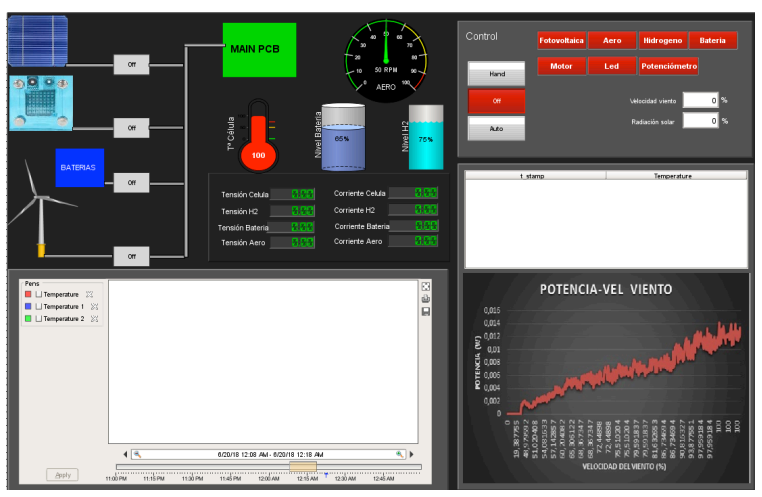

Figura 13: Pantalla control de fuentes.

\subsubsection{Control de cargas}

Es muy similar a la pantalla anterior, pero en este caso es para seleccionar y ver las cargas activas/inactivas Figura 14. 


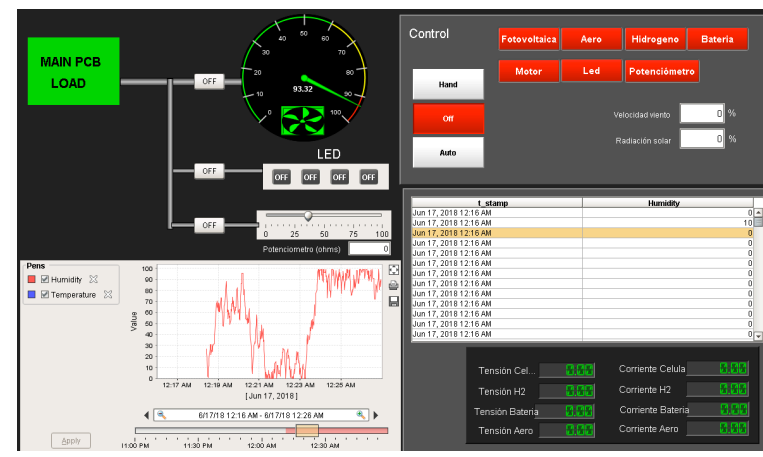

Figura 14: Pantalla cargas.

\subsubsection{Datos de simulación}

En esta pantalla se podrán introducir los valores de radiación de sol, viento o de energía demandada en cada momento, como se ha mencionado anteriormente estos valores pueden ser descargados de los valores reales durante una época del año, así se trabajará con datos reales que se escalaran a un valor adecuado para el sistema.

También se pueden crear simulaciones concretas con variaciones de sol y viento o condiciones determinadas, que se podrán guardar para no tener que introducir todos los valores cada vez, se podrán tener varias simulaciones disponibles con días nublados y vientos débiles o cualquier configuración que se quiera.

\section{$4 \quad$ Funcionamiento del sistema}

Una vez finalizado todo el sistema distribuido, se ha realizado una prueba de funcionamiento donde se han comprobado todos los subsistemas de la planta.

\subsection{Funcionamiento de las comunicaciones}

Primero se ha testeado que las comunicaciones de la planta funcionaran correctamente. Se comienza con la comunicación OPC-UA, que permite la supervisión del sistema mediante el SCADA. Para ello se verifica la comunicación entre la myRIO y el $\mathrm{PC}$, por medio del cliente-servidor OPC-UA, de forma que podemos observar las medidas en el SCADA y ver que se corresponden con el valor en LabVIEW.

Por otro lado, se observa que el valor de radiación y viento que envía LabVIEW llega correctamente a Arduino por medio del protocolo SPI.

\subsection{Funcionamiento del control}

Una vez se ha comprobado que las comunicaciones de la planta son correctas, se pasa al SCADA para realizar otro conjunto de pruebas. Se comprueba que todas las señales de adquisición de datos están obteniendo valores lógicos para las condiciones dadas. Para ello se pondrá el control en modo manual y se comienza a activar cada fuente y carga por separado y se observa los valores. Con esto se comprueba si funciona correctamente la instrumentación de la planta.

Después se verificará el funcionamiento del control de la planta, primero se testea los resultados del regulador Fuzzy en la aplicación de LabVIEW (fuzzy system designer), aquí se puede jugar con los valores de las entradas y verificar que la salida es correcta. Esto se hace con el Test System que aparece en la Figura 15 .

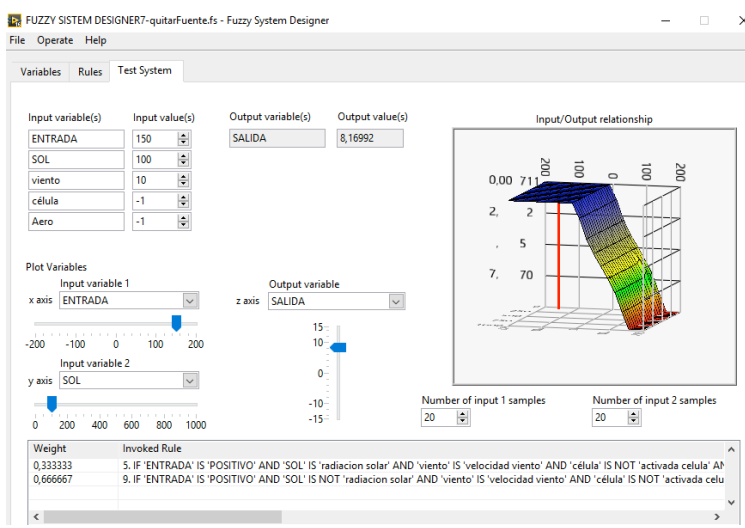

Figura 15: Test controlador Fuzzy.

Después se activa el modo automático desde el SCADA, se introducen los valores de la simulación y se cargarán los datos para comenzar la simulación. La planta deberá ir activando/desactivando las fuentes de energía y almacenamientos de esta (baterías) para satisfacer la demanda de energía marcada.

En las siguientes gráficas se podrá apreciar la salida del regulador que dará un valor entre -10 y 10 , activando la célula fotovoltaica para un valor de entre 0 y 5 , y activa el aerogenerador para un valor mayor a 5, además desactiva las fuentes para el mismo rango de valores en negativo.

En esta prueba se modifica el valor de SetPoint para la corriente que se quiere conseguir, cuando se aumenta el SP aumenta el error (que es la entrada principal al regulador), si se tiene radiación solar o viento y el error es grande se activa una fuente, la fuente a activar la decide el regulador dependiendo del valor de radiación solar, la velocidad del viento y el conjunto de reglas definidas. En las siguientes gráficas se ha usado solo dos fuentes (la célula solar y el aerogenerador) para simplificar la explicación. 
Se realiza la siguiente simulación que se puede observar los resultados en las Figuras 16 y 17, en la que se empieza el test con radiación desde el inicio, por lo que cuando se modifica el SetPoint a un valor de $100 \mathrm{~mA}$ el error hace que aumente la salida del regulador a 3 , con lo que se activa la célula, y la generación de corriente sube hasta unos $90 \mathrm{~mA}$, el error en este punto es pequeño y hace que la salida sea prácticamente cero. Después se aumenta un poco el SetPoint y se comprueba que hasta que el error no aumenta lo suficiente, el regulador no activa la siguiente fuente, el aerogenerador (suponiendo que se tenga suficiente velocidad del viento), ahora la corriente vuelve a subir y se hace el error pequeño de nuevo. Cuando se baja el SetPoint lo suficiente el regulador tendrá que desconectar una fuente, la fuente a elegir depende del valor de radiación y el de velocidad del viento, en este caso la radiación solar se ha bajado a casi el mínimo por lo que se desconecta la célula y el aerogenerador se queda activo. Por último, se vuelve a poner el SetPoint a cero por lo que se desconectan el Aero como se ve al final de la gráfica.

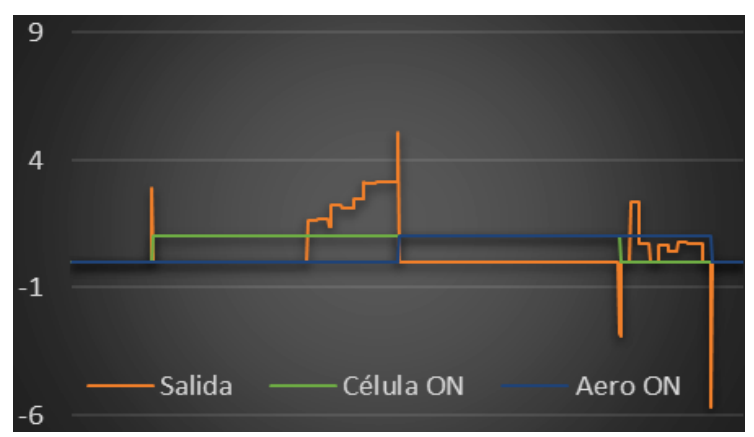

Figura 16: Salida regulador y activación fuentes.

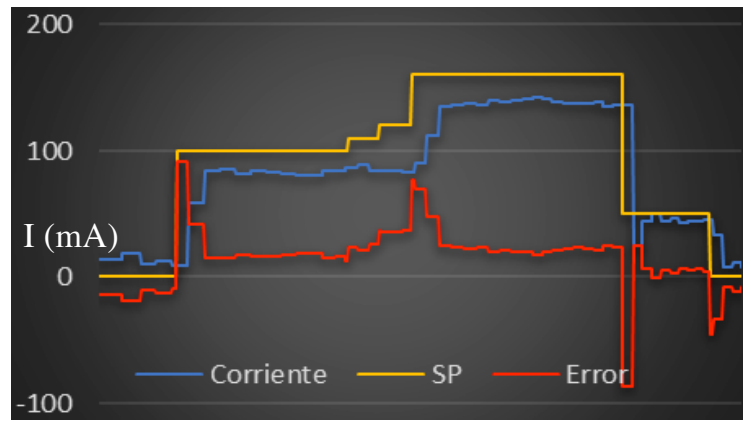

Figura 17: SetPoint, Corriente real y su diferencia.

Como se ha mencionado esta simulación es solo de dos fuentes para simplificar la explicación, pero al añadir la batería se aprovecha mejor la energía, ya que cuando se baja la demanda de energía (SetPoint) en lugar de desconectar la fuente, si hay suficiente radiación o viento, se conecta el modo de carga de batería.

\section{Conclusiones}

Tras llevar a cabo las pruebas anteriores con el sistema en funcionamiento, las conclusiones obtenidas son las siguientes:

- Se ha logrado construir una planta piloto con toda la instrumentación y unas medidas relativamente precisas usando sensores de bajo coste. En los sensores de corriente ha sido donde se ha tenido más dificultad para conseguir valores correctos, debido a la variación entre las medidas consecutivas, por lo que se tuvieron que realizar varias mediciones sobre las que se utilizaba un filtro para obtener valores más precisos.

- Se han programado simulaciones de la planta con diferentes condiciones, tras ajustar los parámetros de velocidad y radiación y hacer diferentes pruebas con todas las fuentes y cargas del sistema.

- Se ha diseñado e implementado un sistema de adquisición y control en tiempo real mediante un regulador difuso, para automatizar el proceso. Esto se lleva a cabo en el hardware myRIO y el programa se implementa en LabView.

- Se ha desarrollado un sistema distribuido perfectamente funcional gracias al uso de los protocolos OPC-UA sobre Ignition y la implementación de un SCADA visual e intuitivo para la supervisión de la planta.

\section{Referencias}

[1] Cayetano Espejo Marín, (1973) La energía solar fotovoltaica en España.

[2] Ernst Friedrich Schumacher, (1973) Lo pequeño es hermoso.

[3] Raquel Gazulla Carceller, TFG: Sistema de caracterización de una pila de hidrógeno a escala. Universitat Politécnica de Valencia

[4] Website:

https://www .arduino.cc/

[5] Website:

http://www.ni.com/es-es/support/model.myrio1900.html

[6] Website:

https://opcfoundation.org

[7] Website:

http://playground .arduino.cc/Main/ACPhaseControl

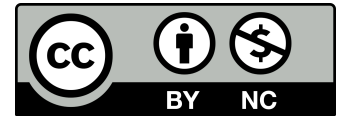

(C) 2018 by the authors. Submitted for possible open access publication under the terms and conditions of the Creative Commons Attribution CC-BY-NC 3.0 license (https://creativecommons.org/licenses/by-nc/3.0). 